\title{
Direct-Coupled Waveguide Filters for the Lower Gigahertz Frequency Range
}

\author{
Vladimir A. Labay, ${ }^{1}$ Jens Bornemann, ${ }^{2}$ Smain Amari, ${ }^{3}$ John M. Damaschke ${ }^{4}$ \\ ${ }^{1}$ Department of Electrical and Computer Engineering, Gonzaga University, Spokane, \\ Washington, USA \\ ${ }^{2}$ Department of Electrical and Computer Engineering, University of Victoria, Victoria, BC, Canada \\ ${ }^{3}$ Department of Electrical and Computer Engineering, Royal Military College, Kingston, Ontario, \\ Canada \\ ${ }^{4}$ Agilent Technologies, Rohnert Park, California, USA
}

Received 25 January 2001; revised 17 August 2001

\begin{abstract}
An analysis method based on the coupled-integral-equations technique (CIET) and the mode-matching technique (MMT) is presented for the design of a variety of directcoupled waveguide filters suitable for applications in the lower gigahertz frequency range. The method is verified by comparison with data obtained through other numerical techniques and measurements. With reference to standard waveguide filters, the issues of filter miniaturization and stopband extension toward higher frequency bands are investigated. For given frequency specifications in the 2-GHz frequency range, examples of rectangular coaxial waveguide filters, ridge waveguide filters, ridge waveguide filters including coupling irises and T-septum waveguide filters are presented. It is demonstrated that the rectangular/square coaxial waveguide filter achieves the highest degree of miniaturization, but that ridge and, especially, T-septum waveguide filter technology is advantageous with respect to stopband performance toward higher frequencies. In particular, a T-septum filter centered at $2.155 \mathrm{GHz}$ is shown to block the entire frequency range up to $7.5 \mathrm{GHz}$. () 2002 Wiley Periodicals, Inc. Int J RF and Microwave CAE 12: 217-225, 2002.
\end{abstract}

Keywords: waveguide technology; filter design; numerical modeling; integral equations; mode-matching techniques

\section{INTRODUCTION}

Direct-coupled resonator waveguide bandpass filters have been used as communication and satellite network components for decades [1]. Accurate synthesis and analysis techniques, many of them based on rigorous field-theoretical methods, are now available to the microwave design engineer, e.g. see ref. 2. In the lower Gigahertz frequency range, however, waveguide filter components are

Correspondence to: Jens Bornemann; e-mail: jbornema@ ece.uvic.ca. usually bulky and exhibit serious shortcomings in operational bandwidth.

This has initiated the development of modified cross-sections such as the ridge waveguide [3], the T-septum waveguide [4-7] and the L-shaped waveguide [8,9]. Although their power-handling capabilities are lower than that of an empty waveguide operated at the same frequency, the gain in operational bandwidth and the potential for miniaturization make these modified waveguides better suited for applications in the lower gigahertz frequency range.

Another frequently used technology consists of square or rectangular coaxial waveguides $[10,11]$ 
that, due to their TEM propagation characteristics, are very well suited for L-band (and lower) feed systems and components, e.g. see ref. 12.

To provide the design engineer with guidelines and performance expectations of filter responses in the lower gigahertz frequency range, this article focuses on a comparison between different direct-coupled waveguide filter technologies. In particular, we investigate the square coaxial waveguide filter, the ridge waveguide filter, the ridge waveguide filter including coupling irises and the T-septum waveguide filter. Although ridge and T-septum structures have been analyzed by members of the group before [13, 14], components have always been interfaced with bulky standard waveguides and have not been used as stand-alone technology. Moreover, for a reliable and accurate mode-spectrum analysis, this article uses the coupled-integral-equations technique (CIET), e.g. see refs. 15 and 16, in the analysis and design process.

\section{THEORY}

For the analysis of filter components in longitudinal (axial) direction, the mode-matching technique (MMT) is used. This procedure is well known, e.g. see ref. 2, and need not be repeated here. Instead we focus on the modespectrum analysis of the individual waveguide cross-sections under consideration, which are illustrated Figure 1. The rectangular waveguide (Fig. 1a) is connected to the rectangular coaxial waveguide (Fig. 1b), the ridge waveguide (Fig. 1c) or the T-septum waveguide (Fig. 1d). For transitions to the rectangular coaxial waveguide, both symmetry planes at $a / 2$ and $b / 2$ are used. Discontinuities involving the ridge and T-septum waveguides employ the symmetry condition at $\mathrm{a} / 2$ only. The most general case is the T-septum waveguide (Fig. 1d) from which the ridge waveguide is derived by letting $g=e$ and eliminating subregion IIc. The rectangular coaxial waveguide (Fig. 1b) is obtained from the ridged waveguide solution by introducing a magnetic wall (m.w.) at $\mathrm{b} / 2$ and adding the solution of the TEM wave to the cross-sectional eigenfunctions.

\section{A. Mode Spectrum}

The TE-mode (subscript $h$ ) cross-section functions, e.g. see ref. 2, for the T-septum waveguide
(Fig. 1d) are given as

$$
\begin{aligned}
T_{h}^{\mathrm{IIa}}= & \sum_{n=0}^{N} A_{n}^{\mathrm{IIa}} \cos \left(k_{x h n}^{\mathrm{IIa}} x\right) \frac{\cos (n \pi y / b)}{\sqrt{1+\delta_{0 n}}}, \\
T_{h}^{\mathrm{IIb}}= & \sum_{k=0}^{K} A_{k}^{\mathrm{IIb}} \frac{\sin \left\{k_{x h k}^{\mathrm{IIb}}\left(x-\frac{a}{2}\right)\right\}}{k_{x h k}^{\mathrm{Ilb}}} \frac{\cos (k \pi y / c)}{\sqrt{1+\delta_{0 k}}}, \\
T_{h}^{\mathrm{IIc}}= & \sum_{l=0}^{L} A_{l}^{\mathrm{IIc}} \cos \left\{k_{x h l}^{\mathrm{IIc}}\left(x-\frac{a}{2}\right)\right\} \\
& \times \frac{\cos [l \pi(y-d) /(b-d)]}{\sqrt{1+\delta_{0 l}}}
\end{aligned}
$$

where $\delta_{0 i}$ is the Kronecker delta and divisions by $k_{x h}$ are introduced to keep the resulting expressions real for imaginary $k_{x h}$. The respective functions for the TM modes (subscript $e$ ) are:

$$
\begin{aligned}
T_{e}^{\mathrm{IIa}}= & \sum_{n=1}^{N} D_{n}^{\mathrm{IIa}} \frac{\sin \left(k_{x e n}^{\mathrm{IIa}} x\right)}{k_{x e n}^{\mathrm{IIa}}} \sin \left(\frac{n \pi}{b} y\right), \\
T_{e}^{\mathrm{IIb}}= & \sum_{k=1}^{K} D_{k}^{\mathrm{IIb}} \cos \left\{k_{x e k}^{\mathrm{IIb}}\left(x-\frac{a}{2}\right)\right\} \sin \left(\frac{k \pi}{c} y\right), \\
T_{e}^{\mathrm{IIc}}= & \sum_{l=1}^{L} D_{l}^{\mathrm{IIc}} \frac{\sin \left\{k_{x e l}^{\mathrm{IIc}}\left(x-\frac{a}{2}\right)\right\}}{k_{x e l}^{\mathrm{IIc}}} \\
& \times \sin \left\{\frac{l \pi}{b-d}(y-d)\right\} .
\end{aligned}
$$

The TEM mode (subscript $T$ ) of the rectangular coaxial waveguide (Fig. 1b) is derived from the equation system of a TM mode with the cutoff wavenumber being zero, e.g. see ref. 10 . Note that under this condition, linear cross-section functions, whose derivatives result in constant fields, are also solutions of the wave equation. With $I_{0}$ being the potential of the center conductor, we get

$$
\begin{aligned}
T_{T}^{\mathrm{IIa}}= & \sum_{n=1}^{N} C_{n}^{\mathrm{IIa}} \sinh \left((2 n-1) \frac{\pi}{b} y\right) \\
& \times \sin \left((2 n-1) \frac{\pi}{b} y\right), \\
T_{T}^{\mathrm{IIb}}= & I_{0} \frac{y}{c}+\sum_{k=1}^{K} C_{k}^{\mathrm{IIb}} \cosh \left(\frac{k \pi}{c} y\right) \sin \left(\frac{k \pi}{c} y\right) .
\end{aligned}
$$

Following the CIET, e.g. see ref. 15, basis functions are introduced, which incorporate the edge 


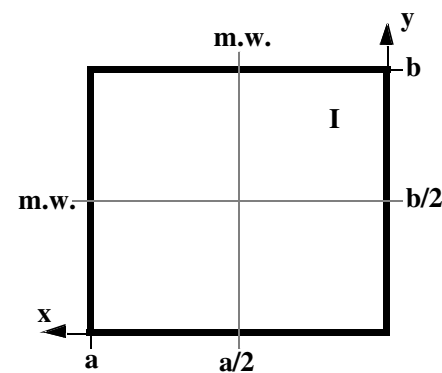

(a)

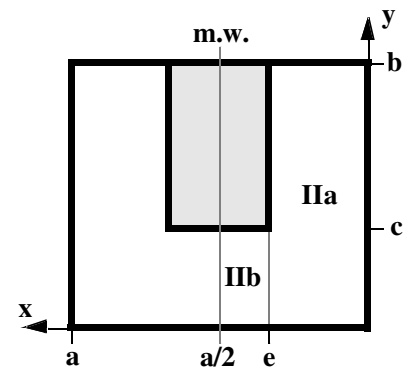

(c)

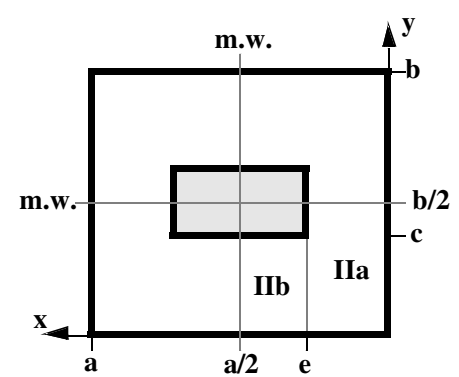

(b)

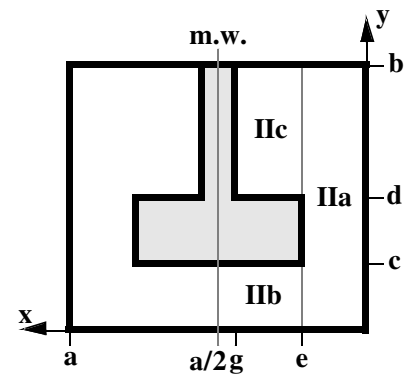

(d)

Figure 1. Cross-sections and symmetry conditions considered in the analysis; (a) rectangular waveguide; (b) rectangular coaxial waveguide; (c) ridged waveguide; (d) T-septum waveguide.

conditions at the respective locations of field singularities as well as their images.

$$
\begin{gathered}
b_{b i}=\frac{\sin [i \pi y / c]}{\sqrt{1+\delta_{0 i}}[(c-y)(c+y)]^{1 / 3}}, \\
b_{c i}=\frac{\cos [(i \pi /(b-d))(y-d)]}{\sqrt{1+\delta_{0 i}}[(d-y)(d-2 b+y)]^{1 / 3}} .
\end{gathered}
$$

Utilizing the Fourier integrals $\widetilde{B}_{b, c}^{\mathrm{IIa}, \mathrm{b}, \mathrm{c}}(n, k, l)$ of the basis functions with respect to adjacent subregions IIa, IIb or IIc, e.g. see ref. 15, and applying Galerkin's method, a homogeneous matrix equation system of the form

$$
\left[\begin{array}{ll}
\underline{A}_{11} & \underline{A}_{12} \\
\underline{A}_{21} & \underline{A}_{22}
\end{array}\right]\left[\begin{array}{l}
\underline{c} \\
\underline{d}
\end{array}\right]=0
$$

is obtained. Here, $c$ and $d$ are the coefficient vectors of basis functions in apertures IIb and IIc, respectively, at $x=e$. For the TE modes of the T-septum waveguide (Fig. 1d), the entries of the submatrices are:

$$
\begin{aligned}
\left(\underline{A}_{11}\right)_{j i}= & \frac{2}{b} \sum_{n=0}^{N} \frac{\cot \left(k_{x h n}^{\mathrm{IIa}} e\right)}{k_{x h n}^{\mathrm{Ia}}} \widetilde{B}_{b j}^{\mathrm{IIa}}(n) \widetilde{B}_{b i}^{\mathrm{IIa}}(n) \\
& -\frac{2}{c} \sum_{k=0}^{K} \frac{\tan \left\{k_{x h k}^{\mathrm{IIb}}((a / 2)-e)\right\}}{k_{x h k}^{\mathrm{IIb}}} \\
& \times \widetilde{B}_{b j}^{\mathrm{IIb}}(k) \widetilde{B}_{b i}^{\mathrm{IIb}}(k),
\end{aligned}
$$

$$
\begin{aligned}
\left(\underline{A}_{12}\right)_{j i}= & \frac{2}{b} \sum_{n=0}^{N} \frac{\cot \left(k_{x h n}^{\mathrm{IIa}} e\right)}{k_{x h n}^{\mathrm{IIa}}} \widetilde{B}_{b j}^{\mathrm{IIa}}(n) \widetilde{B}_{c i}^{\mathrm{IIa}}(n) \\
= & \left(\underline{A}_{21}\right)_{i j}, \\
\left(\underline{A}_{22}\right)_{j i}= & \frac{2}{b} \sum_{n=0}^{N} \frac{\cot \left(k_{x h n}^{\mathrm{IIa}} e\right)}{k_{x h n}^{\mathrm{IIa}}} \widetilde{B}_{c j}^{\mathrm{IIa}}(n) \widetilde{B}_{c i}^{\mathrm{IIa}}(n) \\
& +\frac{2}{b-d} \sum_{l=0}^{L} \frac{\cot \left\{k_{x h l}^{\mathrm{IIc}}(g-e)\right\}}{k_{x h l}^{\mathrm{IIc}}} \\
& \times \widetilde{B}_{c j}^{\mathrm{IIc}}(l) \widetilde{B}_{c i}^{\mathrm{IIc}}(l) .
\end{aligned}
$$

Those of the TM modes are given by:

$$
\begin{aligned}
\left(\underline{A}_{11}\right)_{j i}= & \frac{2}{b} \sum_{n=1}^{N} \frac{k_{x e n}^{\mathrm{IIa}}}{\tan \left(k_{x e n}^{\mathrm{IIa} e} e\right)} \widetilde{B}_{b j}^{\mathrm{IIa}}(n) \widetilde{B}_{b i}^{\mathrm{IIa}}(n) \\
& -\frac{2}{c} \sum_{k=1}^{K} k_{x e k}^{\mathrm{IIb}} \tan \left\{k_{x e k}^{\mathrm{IIb}}\left(\frac{a}{2}-e\right)\right\} \\
& \times \widetilde{B}_{b j}^{\mathrm{IIb}}(k) \widetilde{B}_{b i}^{\mathrm{IIb}}(k), \\
\left(\underline{A}_{12}\right)_{j i}= & \frac{2}{b} \sum_{n=1}^{N} \frac{k_{x e n}^{\mathrm{IIa}}}{\tan \left(k_{x e n}^{\mathrm{IIa}} e\right)} \widetilde{B}_{b j}^{\mathrm{IIa}}(n) \widetilde{B}_{c i}^{\mathrm{IIa}}(n) \\
= & \left(\underline{A}_{21}\right)_{i j},
\end{aligned}
$$




$$
\begin{aligned}
\left(\underline{A}_{22}\right)_{j i}= & \frac{2}{b} \sum_{n=1}^{N} \frac{k_{x e n}^{\mathrm{IIa}}}{\tan \left(k_{x e n}^{\mathrm{IIa}} e\right)} \widetilde{B}_{c j}^{\mathrm{IIa}}(n) \widetilde{B}_{c i}^{\mathrm{IIa}}(n) \\
& +\frac{2}{b-d} \sum_{l=1}^{L} \frac{k_{x e l}^{\mathrm{IIc}}}{\tan \left\{k_{x e l}^{\mathrm{IIc}}(g-e)\right\}} \\
& \times \widetilde{B}_{c j}^{\mathrm{IIc}}(l) \widetilde{B}_{c i}^{\mathrm{IIc}}(l) .
\end{aligned}
$$

The TE- and TM-mode formulations for the rectangular coaxial waveguide (Fig. 1b) and the ridged waveguide (Fig. 1c) follow straightforwardly from the above relations. Only the TEM mode of the coaxial waveguide requires a special treatment. Here we arrive at the inhomogeneous matrix system

$$
[\underline{A}][\underline{c}]=[\underline{D}],
$$

where

$$
\begin{aligned}
(\underline{A})_{j i}= & \frac{4}{b} \sum_{n=1}^{N} \frac{n \pi / b}{\tanh (n \pi e / b)} \widetilde{B}_{b j}^{\mathrm{IIa}}(n) \widetilde{B}_{b i}^{\mathrm{IIa}}(n) \\
& +\frac{4}{c} \sum_{k=1}^{K} \frac{k \pi / c}{\operatorname{coth}(k \pi(a / 2-e) / c)} \\
& \times \widetilde{B}_{b j}^{\mathrm{IIb}}(k) \widetilde{B}_{b i}^{\mathrm{Ilb}}(k) \\
(\underline{D})_{j}= & -I_{0} \frac{2}{b} \sum_{n=1}^{N} \frac{n \pi / b}{\tanh (n \pi e / b)} \\
& \times \widetilde{B}_{b j}^{\mathrm{IIa}}(n) R^{\mathrm{IIa}}(n),
\end{aligned}
$$

and

$$
R^{\mathrm{IIa}}(n)=\left(\frac{b}{n \pi}\right)^{2} \frac{2}{c} \sin (n \pi c / b) .
$$

Note that in all cases, the number of terms in individual subregions appears only as sums in the entries of the respective matrices. Therefore, all entries can be individually tested for convergence with respect to the mode numbers $N, K$, $L$. The matrix size depends exclusively on the number of edge-conditioned basis functions. This is one of the salient features of the CIET formulation as problems associated with relative convergence are eliminated.

\section{B. Modal Scattering Matrix}

The computation of the modal scattering matrix from an empty waveguide (Fig. 1a) to one of the three cross-sections in Figure 1(b)-(d) follows the general mode-matching technique, e.g. see ref. 2. Modes are arranged with respect to TEM, TE and
TM modes and increasing cutoff frequencies, the lowest being the TEM mode (Fig. 1b) and the $\mathrm{TE}_{1}$ mode for the structures in Figure 1(c), and (d). Therefore, the coupling matrix is arranged as

$$
\underline{M}=\left[\begin{array}{ccc}
\underline{0} & \underline{J}_{h h} & \underline{0} \\
\underline{J}_{e T} & \underline{J}_{e h} & \underline{J}_{e e}
\end{array}\right],
$$

where the number of columns is given by the mode spectrum of the coaxial waveguide and the number of rows by that of the empty waveguide. Note that $\underline{J}_{e T}$ is a single-column matrix, which disappears if the TEM mode is not present, e.g., for ridged waveguide and T-septum structures. The entries of submatrices $\underline{J}$ in (22) are given by

$$
\begin{aligned}
& \left(\underline{J}_{h h}\right)_{q r}=\int_{A^{\mathrm{II}}}\left[\nabla T_{h q}^{\mathrm{I}} \nabla T_{h r}^{\mathrm{II}}\right] d A, \\
& \left(\underline{J}_{e T}\right)_{p}=\int_{A^{\mathrm{II}}}\left[\nabla T_{e p}^{\mathrm{I}} \nabla T_{T}^{\mathrm{II}}\right] d A, \\
& \left(\underline{J}_{e h}\right)_{p r}=-\int_{A^{\mathrm{II}}}\left[\nabla T_{e p}^{\mathrm{I}}\left(\nabla T_{h r}^{\mathrm{II}} \times \vec{u}_{z}\right)\right] d A, \\
& \left(\underline{J}_{e e}\right)_{p s}=\int_{A^{\mathrm{II}}}\left[\nabla T_{e p}^{\mathrm{I}} \nabla T_{e s}^{\mathrm{II}}\right] d A,
\end{aligned}
$$

where $T_{h q}^{\mathrm{I}}, T_{e p}^{\mathrm{I}}$ denote the well-known TE-, TM-mode cross-section functions (c.f. [2]), respectively, of the empty waveguide $I$ (Fig. 1a), and $\vec{u}_{z}$ is the unit vector in axial direction.

Once the modal scattering matrix of a single discontinuity is determined, filter structures can be analyzed by cascading waveguide sections and modal scattering matrices of subsequent discontinuities. To verify the approach presented here, results obtained by this technique are compared with data available from the literature and/or measurements. Excellent agreement is demonstrated in Figure 2 for a rectangular coaxial waveguide filter and in Figure 3 for a configuration involving ridge waveguide discontinuities.

\section{RESULTS}

In this section, we compare a number of different filter configurations for similar design specifications. A typical design cycle includes a filter synthesis using impedance/admittance inverters, e.g. see ref. 2, and a fine optimization involving minimax algorithms [17].

Filter comparisons can be carried out by maintaining certain parameters and improving others. Usually, bandwidth and return loss are kept constant whereas the topology can vary. In this case, 


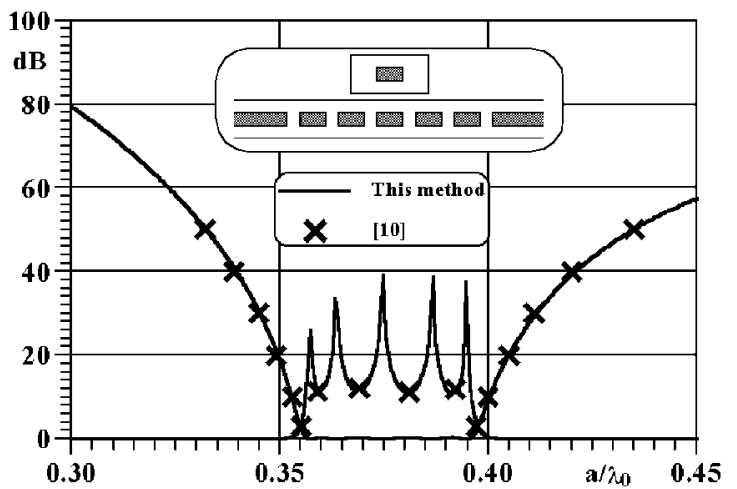

Figure 2. Response of a five-pole rectangular coaxial waveguide filter and comparison with data presented in [10].

we opted for maintaining bandwidth and the number of resonators. The reason behind this approach is the relationship between return loss, stopband performance and degree of achievable miniaturization for some of the structures involved. Moreover, the stopband performance (both close to the passband and toward higher frequencies) is related to the number of resonators (return loss poles). Therefore, filter designs are carried out for a given number of six resonators while achieving a reasonable compromise between stopband attenuation and inband return loss. Emphasis is placed on the overall filter size, namely the inner cross-section dimensions of the waveguide housing and filter length measured from the first to the last coupling section (the usual matching sections to coaxial cable are excluded) and the stopband behavior toward higher frequencies.

As the first example, a standard waveguide inductive-iris filter is designed for a passband of 2.108-2.205 GHz, 27-dB return loss and stopband specifications of $30 \mathrm{~dB}$ at 2.07 and 2.27

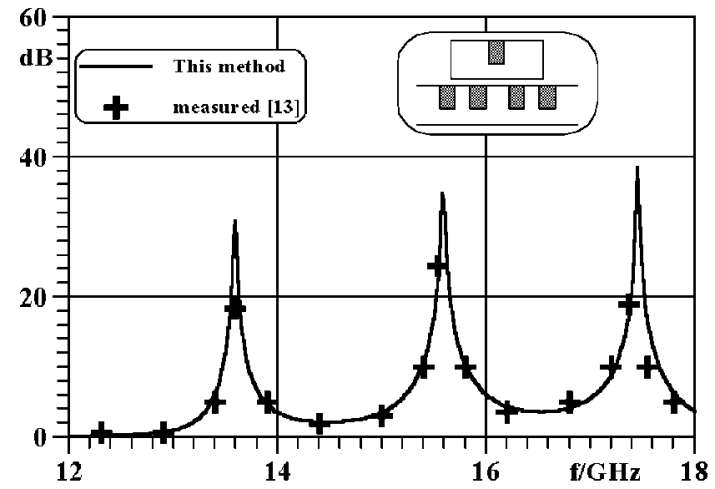

Figure 3. Return loss of four ridged sections in $\mathrm{Ku}$-band waveguide and comparison with measurements in [13].
GHz. The performance of the resulting six-pole filter is depicted in Figure 4. Due to dispersion in standard waveguide filters, the second passpand (2.94-3.23 GHz, 30-dB stopband up to $2.86 \mathrm{GHz}$ ) occurs far below the theoretical harmonic passband of 4.216-4.41 GHz. Moreover, and especially due to the application in the lower gigahertz frequency range, the filter is quite bulky with inner dimensions of $86.36 \mathrm{~mm} \times 43.18 \mathrm{~mm} \times$ $515.92 \mathrm{~mm}=1.99 \times 10^{6} \mathrm{~mm}^{3}$.

The investigation now focuses on reducing the overall filter size and improving the poor stopband performance of the standard waveguide filter in Figure 4.

In the frequency range of interest, rectangular/square coaxial waveguide technology is commonly employed in satellite feed systems [12]. As described in the previous section, the mode spectrum of a square coaxial cross-section with $50 \Omega$ impedance is analyzed using ten basis functions and 50 expansion terms. The mode-matching technique for the rectangular-to-coaxial waveguide discontinuity utilizes up to 84 modes altogether. (Similar numbers are used for the analysis and design of all filters presented in this section.) After initial synthesis and optimization, the filter performance shown in Figure 5 is obtained. Compared with the filter of Figure 4, the inband return loss is now $34 \mathrm{~dB}$, the stopband attenuation level is significantly increased, and the second passband agrees with the harmonic one as expected for TEM propagation. The filter size is $15.24 \mathrm{~mm} \times$ $15.24 \mathrm{~mm} \times 394.6 \mathrm{~mm}=9.16 \times 10^{4} \mathrm{~mm}^{3}-$ a size reduction by more than a factor of 20 . Depending on the power level to be handled by this filter, a further size reduction is possible with respect to the cross-section. A similar filter design (not shown here) uses a $7.62 \mathrm{~mm}$ square housing and

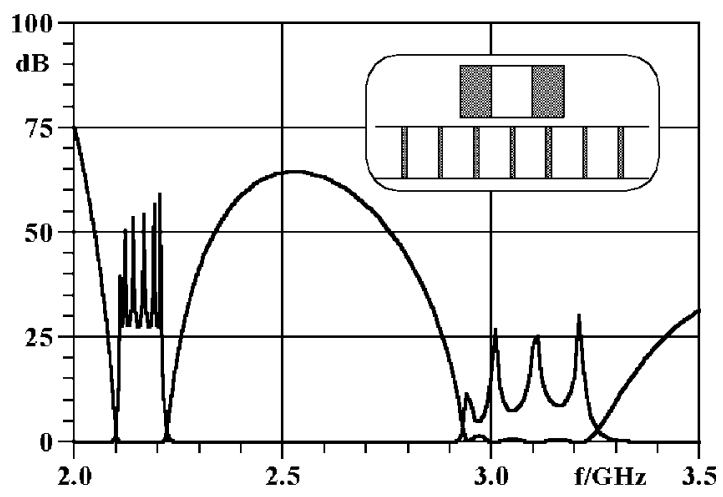

Figure 4. Insertion and return loss of synthesized/optimized six-pole inductive-iris waveguide filter for $2.155 \mathrm{GHz}$. 


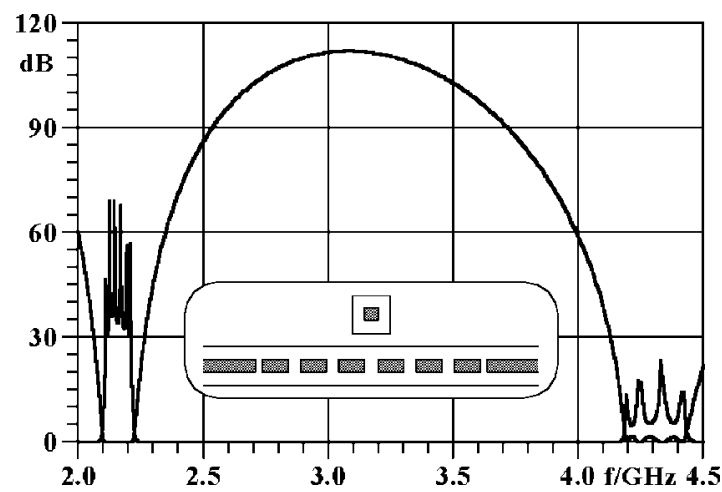

Figure 5. Insertion and return loss of synthesized/optimized six-pole square coaxial waveguide filter for $2.155 \mathrm{GHz}$.

occupies an inner space of $2.26 \times 10^{4} \mathrm{~mm}^{3}$, thus resulting in a reduction factor of 88 .

A further improvement of the stopband performance is possible by utilizing a waveguide technology in which modifications to the standard rectangular cross-section allow for the fundamental-mode cutoff frequency to be reduced substantially. A typical example is the ridge wave-

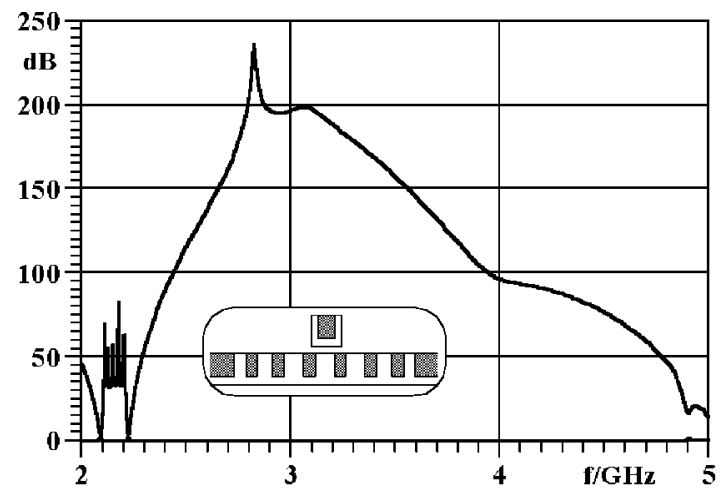

(a)

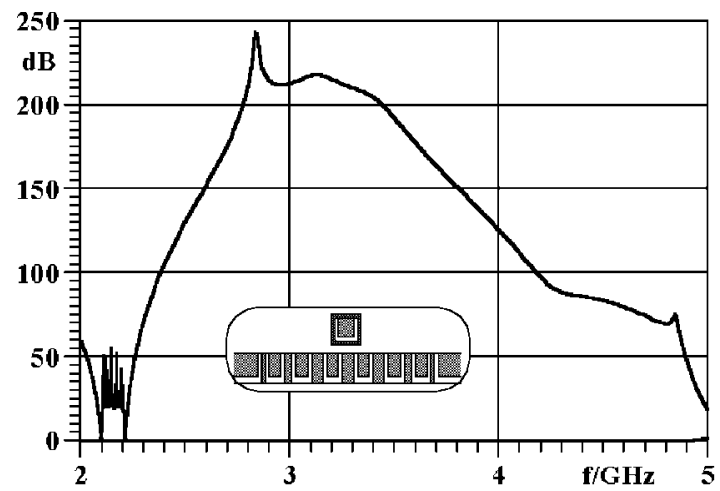

(b)

Figure 6. (a) Insertion and return loss of synthesized/optimized six-pole ridge waveguide filter for $2.155 \mathrm{GHz}$. (b) Performance of six-pole ridge waveguide filter with reduced-size waveguides in coupling sections. guide and Figure 6(a) presents the response of such a filter design. The inband return loss is 32 $\mathrm{dB}$ and the $30-\mathrm{dB}$ stopband is extended to 4.86 $\mathrm{GHz}$. The inside filter dimensions are $38.1 \mathrm{~mm}$ $\times 38.1 \mathrm{~mm} \times 258.44 \mathrm{~mm}=37.5 \times 10^{4} \mathrm{~mm}^{3}$, which is larger than the square coaxial design yet more than a factor of 5 smaller than the standard waveguide filter of Figure 4.

Some discussions were focused on a further size reduction of the ridge waveguide filter by introducing smaller waveguide sections between the ridge resonators. The data associated with Figure 6(b), however, prove this assumption wrong. The filter performance is improved only slightly and so is the overall size of this filter $\left(38.4 \times 10^{4} \mathrm{~mm}^{3}\right)$, which uses the same crosssection as that in Figure 6(a). The reason for this is that in Figure 6(a), the resonators are much shorter than half a wavelength due to the highly evanescent character of the below-cutoff sections between the ridges [18]. With reduced size waveguide sections between the ridges, however, the iris character of such sections encourages the forma-

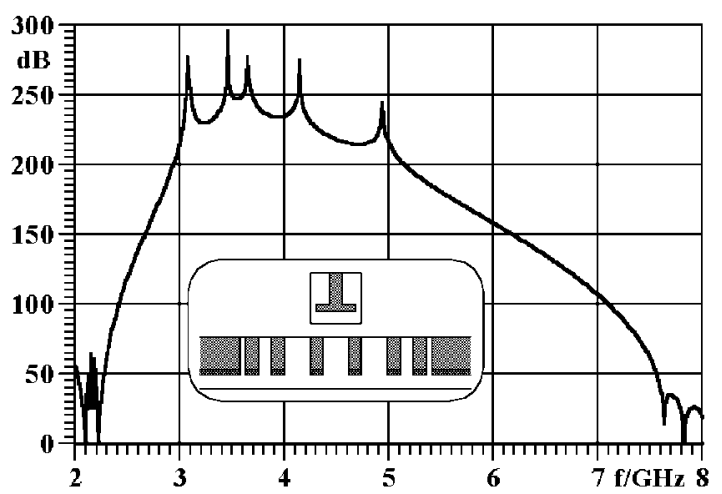

(a)

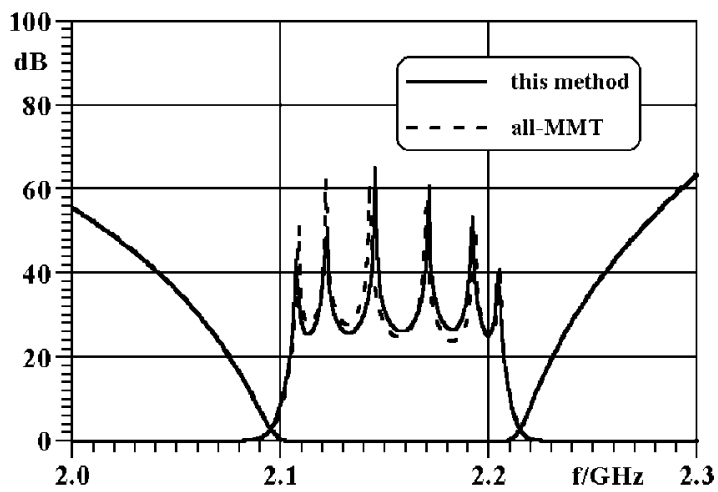

(b)

Figure 7. (a) Insertion and return loss of synthesized/optimized six-pole T-septum waveguide filter for $2.155 \mathrm{GHz}$. (b) Validation of T-septum filter design by independently developed all-MMT software. 
TABLE I. Performance Comparison of Six-Resonator 2.155-GHz Waveguide Filters

\begin{tabular}{lcccc}
\hline Filter Type & $\begin{array}{c}\text { (Achievable) } \\
\text { Return Loss [dB] }\end{array}$ & $\begin{array}{c}\text { Attenuation } \\
\text { up to }\{\mathrm{GHz}\}\end{array}$ & $\begin{array}{c}\text { Size Reduction } \\
\text { to Waveguide } \\
\text { Iris Filter }\end{array}$ & Figure \\
\hline Waveguide iris & 27 & 2.86 & 1 & 4 \\
Square coax & 34 & 4.13 & $20-88$ & 5 \\
Ridged waveguide & 32 & 4.86 & 5.3 & $6(\mathrm{a})$ \\
$\begin{array}{l}\text { Aperture-coupled } \\
\quad \text { ridged waveguide }\end{array}$ & 20 & 4.95 & 5.7 & $6(\mathrm{~b})$ \\
T-septum waveguide & 24 & 7.60 & 27 & 7 (a) and (b) \\
\hline
\end{tabular}

tion of close to half-wavelength ridge resonators, which makes the performance similar to that of the standard waveguide filter in Figure 4. Once the optimization is set to enforce the stopband up to $4.86 \mathrm{GHz}$, the structure of Figure 6(b) does not offer a real alternative to that of Figure 6(a), especially not so with respect to manufacturing issues and a reduced inband return loss of $20 \mathrm{~dB}$.

The last technology investigated is the $\mathrm{T}$ septum waveguide, e.g. see refs. 7, and 14 . Figure 7(a) shows the performance of a comparable T-septum waveguide filter. The inband return loss is $24 \mathrm{~dB}$, and the $30-\mathrm{dB}$ stopband is pushed up to $7.6 \mathrm{GHz}$. The filter dimensions are $25.4 \mathrm{~mm}$ $\times 19.05 \mathrm{~mm} \times 147.45 \mathrm{~mm}=7.13 \times 10^{4} \mathrm{~mm}^{3}$, which brings this design in the order of magnitude of a square coaxial waveguide filter, although with a stopband performance that cannot be achieved by directly coupled coaxial resonators. Also shown (Fig. 7b) are the results of an independently developed code using the mode-matching technique without incorporating edge conditions (all-MMT). Except for some minor deviations beyond 23.5-dB return loss, the agreement between the two methods is excellent, thus validating the theoretical approach presented here.

Table I compares the performances of the 2.155-GHz filters designed with the technique presented in this article. From a view of good return loss and very high degree of miniaturization, the square coaxial filter appears to be the obvious choice. However, T-septum filter technology achieves by far the best stopband performance even while maintaining a small size and reasonable return loss.

Finally, we would like to address the issue of losses. From the theory presented, it is apparent that the analysis assumes all conducting material to be ideally conducting. Whereas a loss analysis can straightforwardly be included in standard waveguide filters (e.g., a silver-plated version of the filter in Figure 4 will exhibit 0.12 -dB insertion loss), this is extremely difficult for the structures presented in Figures 6 and 7. Following a pertubation technique applied to a T-septum waveguide filter in a diplexer configuration [19], it is assumed that the T-septum filter of Figure 7, when silver plated, will be measured with approximately 1.5-dB insertion loss. Whether this can be justified in applications requiring the extended stopband and associated miniaturization, remains to be decided on an individual basis.

\section{CONCLUSIONS}

A variety of different waveguide filter configurations is presented for an application in the $2-\mathrm{GHz}$ frequency range. As expected, the rectangular/square coaxial waveguide filter is the preferred choice if miniaturization is the dominant design criterion. However, if extended stopband performance together with a suitable degree of miniaturization are the main requirements, then the T-septum waveguide filter or even the ridge waveguide filter presents powerful alternatives to coaxial-waveguide and standard-waveguide technology. The theoretical analysis technique is verified for a number of different configurations and, therefore, inspires confidence in the design data presented.

\section{REFERENCES}

1. G.L. Matthaei, L. Young, and E.M.T. Jones, Microwave filters, impedance matching networks and coupling structures, Artech House, Dedham, 1980.

2. J. Uher, J. Bornemann, and U. Rosenberg, Waveguide components for antenna feed systems: theory and CAD, Artech House, Norwood, 1993. 
3. S. Hopfer, The design of ridged waveguides, IRE Trans Microwaves Theory Tech MTT-3 (1955), 20-29.

4. Y. Zhang and W.T. Jones, Attenuation and powerhandling capability of T-septum waveguides, IEEE Trans Microwave Theory Tech MTT-35 (1987), 858-861.

5. B.E. Pauplis and D.C. Power, On the bandwidth of T-septum waveguide, IEEE Trans Microwave Theory Tech 37 (1989), 919-922.

6. F.J. German and L.S. Riggs, Bandwidth properties of rectangular T-septum waveguides, IEEE Trans Microwave Theory Tech 37 (1989), 917-919.

7. P.K. Saha and G.G. Mazumder, Bandwidth characteristics of inhomogeneous T-septum waveguides, IEEE Trans Microwave Theory Tech 37 (1989), 1021-1026.

8. P.K. Saha and D. Guha, New broadband rectangular waveguide with L-shaped septa, IEEE Trans Microwave Theory Tech 40 (1992), 777-781.

9. P.K. Saha and D. Guha, Bandwidth and dispersion characteristics of a new rectangular waveguide with two L-shaped septa, IEEE Trans Microwave Theory Tech 47 (1999), 87-92.

10. G.-U. Paul, Exact calculation of microwave filters composed of rectangular transmission line resonators (in German), Frequenz 30 (1976), 335-339.

11. L. Gruner, Higher order modes in square coaxial lines, IEEE Trans Microwave Theory Tech MTT-31 (1983), 770-772.
12. F. Alessandri, M. Mongiardo, and R. Sorrentino, Computer-aided design of beam forming networks for modern satellite antennas, IEEE Trans Microwave Theory Tech 40 (1992), 1117-1127.

13. J. Bornemann and F. Arndt, Modal S-matrix design of metal finned waveguide components and its application to transformers and filters, IEEE Trans Microwave Theory Tech 40 (1992), 1528-1537.

14. V.A. Labay and J. Bornemann, CAD of T-septum waveguide evanescent-mode filters, IEEE Trans Microwave Theory Tech 41 (1993), 731-733.

15. S. Amari, J. Bornemann, and R. Vahldieck, Application of a coupled-integral-equations technique to ridged waveguides, IEEE Trans Microwave Theory Tech 44 (1996), 2256-2264.

16. S. Amari, J. Bornemann, and R. Vahldieck, Fast and accurate analysis of waveguide filters by the coupled-integral-equation technique, IEEE Trans Microwave Theory Tech 45 (1997), 1611-1618.

17. K. Madsen, H. Schaer-Jacobsen, and J. Voldby, Automated minimax design of networks, IEEE Trans Circuits Systems CAS-22(1997), 791-796.

18. S. Amari, J. Bornemann, and R. Vahldieck, A technique for designing ring and rod dielectric resonators in cutoff waveguides, Microwave Opt Technol Lett 23(4) (1999), 203-205.

19. V.A. Labay, Computer-aided design of passive microwave components in nonstandard rectangular waveguide technology, Ph.D. Dissertation, University of Victoria, Victoria, BC, Canada, 1995.

\section{BIOGRAPHIES}

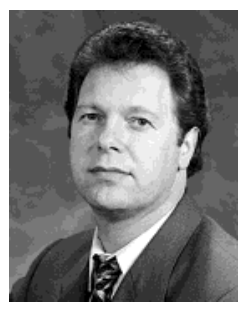

Jens Bornemann received the Dipl.-Ing. and the Dr.-Ing. degrees, both in Electrical Engineering, from the University of Bremen, Germany, in 1980 and 1984, respectively. From 1984 to 1985 , he was a consulting engineer. In 1985, he joined the University of Bremen, Germany, as an Assistant Professor. Since April 1988, he has been with the Department of Electrical and Computer Engineering, University of Victoria, Victoria, B.C., Canada, where he became a Professor in 1992. From 1992 to 1995, he was a Fellow of the British Columbia Advanced Systems Institute. In 1996, he was a Visiting Scientist at Spar Aerospace Limited (now EMS Technologies Inc.), Ste-Anne-de-Bellevue, Québec, Canada, and a Visiting Professor at the Microwave Department, University of Ulm, Germany. Since 1997, he has been a co-director of the Center for Advanced Materials and Related Technology (CAMTEC), University of Victoria. He has coauthored
Waveguide Components for Antenna Feed Systems. Theory and Design (Norwood, MA: Artech House, 1993) and has authored/coauthored more than 170 technical articles. His research activities include microwave/millimeter-wave components and systems design, and problems involving electromagnetic field theory in integrated circuits, waveguide feed networks and radiating structures. He serves on the editorial advisory board of the International Journal of Numerical Modelling. Dr. Bornemann is a Registered Professional Engineer in the Province of British Columbia, Canada. He is a Senior Member of IEEE Microwave Theory and Techniques and Antennas and Propagation Societies. He was a co-recipient of the A.F. Bulgin Premium of the Institution of Electronic and Radio Engineers in 1983. Currently, he serves as an Associate Editor of the IEEE Transactions on Microwave Theory and Techniques in the area of Microwave Modeling and CAD and on the Technical Program Committee of the IEEE MTT-S International Microwave Symposium. 


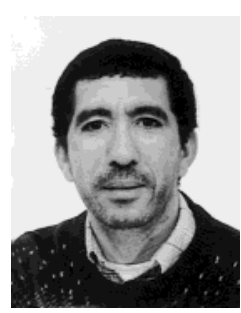

Smain Amari received the D.E.S degree in physics and electronics from the University of Constantine (Algeria) in 1985, the Master's degree in Electrical Engineering in 1989 and the $\mathrm{PhD}$ degree in physics in 1994 both from Washington University in St.Louis. From May 1994 to November 2000, he was with the Department of Electrical and Computer Engineering at the University of Victoria, Canada, as a postdoctoral fellow, research associate and adjunct assistant professor. He joined the department of Electrical and Computer Engineering of the Royal Military College of Canada in Kingston in November 2000 as an Assistant Professor. He was a visiting scientist and visiting professor at the Swiss Federal Institute of Technology in Zurich, Switzerland. He is interested in numerical analysis and modeling, applied mathematics and applied physics, electromagnetics and microwaves, and quantum many-body problems.

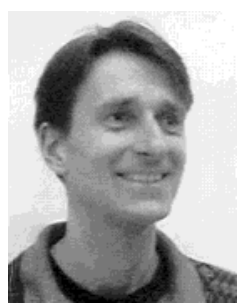

John M. Damaschke was born in 1968 in Hamburg, Germany. He received his Vordiplom (B.Sc.) and Dipl.-Ing. (M.A.Sc.) in Electrical Engineering from the Technical University HamburgHarburg in 1990 and 1994, respectively. In 1993, he worked in Power Electronics in the Department of Electrical Engineering at the University of Manchester, GB. From 1993 to 2000 he was employed in the Department of Electrical Engineering at the University of
Victoria, Canada, where he specialized in numerical modeling in electromagnetics. Since 2000, he has been working for a major manufacturer of measurement equipment in California.

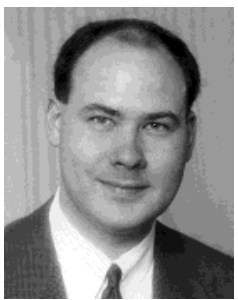

Dr. Vladimir Labay is currently Assistant Professor at Gonzaga University in Spokane, Washington, USA. In 1987 and 1990 Dr. Labay received a B.Sc.(E.E.) and M.Sc.(E.E.), respectively, from the University of Manitoba, Winnipeg, MB. After graduating with a Ph.D. in 1995, he remained at the University of Victoria, Victoria, $\mathrm{BC}$ as a lecturer and research engineer until he accepted an assistant professor position at Eastern Washington University in Cheney, WA, USA. Before joining Gonzaga University, Dr. Labay also held positions of visiting professor at the University of Idaho, Moscow, ID and of adjunct professor at Washington State University, Pullman, WA. His research interests include the development of computer-aided design software for microwave wave-break guide components used in wireless and satellite communications. 